\title{
HIV-related morbidity rate, thirteen years after the introduction of highly active antiretroviral therapy (1996-2009)
}

Roberto Manfredi

From $16^{\text {th }}$ International Symposium on HIV and Emerging Infectious Diseases

Marseille, France. 24-26 March 2010

\section{Background}

After the availability of combined antiretroviral therapy (cART), we quantified the consequences on the general morbidity rates, and HIV-related hospitalization rates, in the period 1992-2008.

\section{Methods}

HIV-associated hospitalizations were assessed according to three different periods of time: before cART introduction (1992-1995), immediately after first cART availability (1996-1998), and the last one, referred to the fully established cART era (1999-2008).

\section{Results}

During the three examined periods, an undetectable viremia was never detected in any patient in the pre-cART era, in $21 \%$ of cases in the first years of cART, and in $41 \%$ of patients in the last years of cART $(p<.0001)$. In parallel, the mean CD4+ T-lymphocyte count in the three study groups tested $27.2 \pm 11.3$ cells $/ \mu \mathrm{L}, 39.3 \pm 14.6$ cells/ $\mu \mathrm{L}$, and $89.6 \pm 38.2$ cells $/ \mu \mathrm{L}$, respectively $(p<.001)$. During time, an increased frequency of hospitalization of heterosexual and female patients occurred, while the frequenct of IVDA had a significant drop (from $69 \%$ in the pre-cART period, to $57 \%$ during initial cART era, to $39 \%$ at the time of consolidated cART era; $p<.0001)$. The patients with a prior diagnosis of full-blown AIDS represented $86 \%, 57 \%$, and $33 \%$, respectively $(p<.0001)$, while hospitalized inpatients who experienced a diagnosis of AIDS concurrently with the first detection of HIV infection (the so-called "AIDS presenters"), showed an evident temporal increase $(11 \%, 21 \%$, and $39 \%$, respectively; $p<.0001)$. Among concurrent illnesses, a huge rise of chronic liver diseases was registered from the pre-cART time (18\%), to the first years of cART availability (29\%), to the current time of advanced cART $(48 \%)(p<.001)$, while an increased mortality due to hematological and solid malignancies also occurred, although at a lesser extent $(8.2 \%, 11.7 \%$, and $17.8 \%$ respectively; $p<.001)$.

\section{Discussion}

The introduction of cART profoundly acted on the general morbidity for HIV infection and AIDS, although the epidemiological-clinical-laboratory scenario significantly changed over time. These modifications need a careful monitoring, in order to ensure a timely diagnostic and clinical disease recognition by all involved health caregivers who face HIV-infected patients, and to plan an adequate allocation of available resources, funding, structures, and dedicated personnel.

Published: 11 May 2010

doi:10.1186/1742-4690-7-S1-P33

Cite this article as: Manfredi: HIV-related morbidity rate, thirteen years after the introduction of highly active antiretroviral therapy (19962009). Retrovirology 2010 7(Suppl 1):P33.

Correspondence: Roberto.manfredi@unibo.it

Infectious Diseases, University of Bologna, S. Orsola Hospital, Bologna, Italy 